\title{
Region-specific distribution of Olig2-expressing astrocytes in adult mouse brain and spinal cord
}

\author{
Hui Wang ${ }^{1,2}$, Liang Xu3 ${ }^{3}$, Chuying Lai ${ }^{3}$, Kaiyu Hou ${ }^{3}$, Junliang Chen ${ }^{3}$, Yaowei Guo ${ }^{3}$, Abhijeet Sambangi ${ }^{2}$, \\ Shreya Swaminathan ${ }^{2}$, Chunming $\mathrm{Xie}^{1,4^{*}} \mathbb{0}$, Zheng Wu $\mathrm{u}^{2,3^{*}}$ and Gong Chen ${ }^{2,3^{*}}$
}

\begin{abstract}
Olig2 is an important transcription factor essential for the specification and differentiation of oligodendrocytes as well as astrocytes and neurons during developmental stages. However, Olig2 distribution pattern and its relationship among different types of glial cells in the adult central nervous system (CNS) are not well characterized. Here, we systematically examined Olig2 expression pattern in combination with major markers of neurons and glial cells throughout the brain and spinal cord in the adult mice. As expected, Olig2 is universally expressed in oligodendrocytes and oligodendrocyte precursor cells (OPCs), but not in neurons or microglia. Interestingly, we discover a subpopulation of Olig2 ${ }^{+}$astrocytes that are highly enriched in some specific regions including the olfactory bulb, thalamus, midbrain, medulla, and spinal cord in the adult mice. Moreover, OPCs have high expression level of Olig2, whereas oligodendrocytes and astrocytes have similar level of Olig2 expression. Our results suggest that a distinct population of Olig2 ${ }^{+}$ astrocytes are highly concentrated in discrete regions in the adult CNS. Investigating the functional significance of these Olig2 ${ }^{+}$astrocytes in both resting state and pathological state of the brain and spinal cord may broaden our understanding on astrocytic heterogeneity and functions.
\end{abstract}

Keywords: Olig2, Astrocyte, Oligodendrocyte, Neuron, Central nervous system, Brain, Spinal cord, Microglia

\section{Introduction}

Oligodendrocyte transcription factor 2 (Olig2) is a basic helix-loop-helix (bHLH) transcription factor that is mainly expressed in the CNS [1]. Olig2 regulates neurogenesis primarily as an anti-neurogenic factor but may also be required for certain neuronal differentiation at different stages of development [2,3]. During embryogenesis, Olig2 first guides the fate of motor neurons by establishing the ventral domain of motor neuron

*Correspondence: chmxie@163.com; zhengwu@jnu.edu.cn; gongchen@jnu. edu.cn

${ }^{1}$ Department of Neurology, Affiliated ZhongDa Hospital, School of Medicine, Southeast University, Nanjing 210009, Jiangsu, China

2 Department of Biology, Huck Institutes of Life Sciences, Pennsylvania State University, University Park, PA 16802, USA

Full list of author information is available at the end of the article ancestors and promoting neuronal differentiation, and then switches to promoting the formation of OPCs and the differentiation of oligodendrocytes in later stages of development [4-6]. Recent studies have found that Olig2 is expressed in developing astrocytes and plays a pivotal role in astrocyte development in the brain [7-10]. These studies indicate that Olig2 acts as a multifaceted transcription factor for some specific cell type differentiation and maturation during neural development. In the normal adult CNS, Olig2 is continuously expressed in the OPCs, mature oligodendrocytes [11] and a very small percentage of astrocytes ( 4.3\%) [12]. Interestingly, the postnatal deletion of Olig2 will switch OPCs to an astrocyte fate [13]. Olig2 can also be found in reactive astrocytes after brain injury [14] and plays a critical role in reactive astrocyte proliferation and glial scar formation [15]. In general, Olig2 ${ }^{+}$OPCs can differentiate original author(s) and the source, provide a link to the Creative Commons licence, and indicate if changes were made. The images or other third party material in this article are included in the article's Creative Commons licence, unless indicated otherwise in a credit line to the material. If material is not included in the article's Creative Commons licence and your intended use is not permitted by statutory regulation or exceeds the permitted use, you will need to obtain permission directly from the copyright holder. To view a copy of this licence, visit http://creativecommons.org/licenses/by/4.0/. The Creative Commons Public Domain Dedication waiver (http://creativeco mmons.org/publicdomain/zero/1.0/) applies to the data made available in this article, unless otherwise stated in a credit line to the data. 
into oligodendrocytes during adulthood [11]. A previous study even reported that OPCs in the piriform cortex can generate some piriform pyramidal neurons in the adult brain [16]. Therefore, Olig2 can be expressed in different cell types and play diversified functions in the adult CNS. However, considering the heterogeneity of cell composition among different CNS regions, the precise Olig2 expression pattern in the adult CNS remains poorly understood.

To systematically investigate the Olig2 expression pattern in the adult mouse CNS, we performed a series of studies on Olig2 expression in the adult CNS by investigating five major subtypes of cells including OPCs, oligodendrocytes, astrocytes, microglia, and neurons. We found that almost all the OPCs and oligodendrocytes express Olig2. No Olig2 signal was detected in microglia or neurons. Interestingly, the expression pattern of Olig2 in adult astrocytes shows region-specific patterns. Most astrocytes in the adult mouse cortex are Olig2-negative. Surprisingly, over $80 \%$ of astrocytes are Olig $2^{+}$in a few regions including the olfactory bulb, midbrain, thalamus, medulla and the spinal cord. Furthermore, the expression level of Olig2 appears to be much higher in OPCs than that in oligodendrocytes and astrocytes. Together, our study provides a holistic view of Olig2 distribution in the adult mouse brain and spinal cord. Our discovery of several discrete CNS regions with high level of Olig2-expression astrocytes suggests that these Olig2 ${ }^{+}$astrocytes may play a distinct function from those Olig2 ${ }^{+}$astrocytes.

\section{Results}

\section{NG2-expressing cells are only a proportion}

\section{of Olig2-expressing cells}

OPCs are a population of glial cells in the CNS which comprise about $5 \%$ of total cells and are the main proliferating cell type in the resting state of adult CNS [11]. In the adult CNS, OPCs can differentiate into myelinating oligodendrocytes and are usually characterized as NG2 proteoglycan expressing cells. Therefore, we performed co-immunostaining of Olig2 and NG2 to study their relative distributions in the adult mouse CNS. Figure 1 illustrates the overlay of both Olig2 and NG2 signals across different brain regions (Fig. 1a, sagittal brain section at low magnification) and the spinal cord (SC) (Fig. 1n, coronal section) in an adult mouse. Clearly, while Olig2 and NG2 signal can be detected throughout the CNS regions, their relative expression level is not evenly distributed among different regions. One striking feature is the obvious strong signal of Olig2 (green) in the thalamus $(\mathrm{TH})$ and midbrain (MB) (Fig. 1a). To more clearly understand the relationship between the NG2 and Olig2 expression pattern, we divided the brain into 12 discrete regions including the olfactory bulb $(\mathrm{OB})$, anterior olfactory nucleus (AON), motor cortex (MO), striatum (STR), somatosensory cortex (SS), visual cortex (VIS), retrosplenial area (RSP), hippocampus (Hip), thalamus (TH), midbrain (MB), cerebellum (CB), and medulla (MY) (Fig. 1a). As shown in the high magnification confocal images of the brain (Fig. 1b-m) and SC (Fig. 1o), almost all the NG2-expressing cells in different CNS regions were clearly co-stained with Olig2, suggesting that all NG2 cells express Olig2. However, there were a considerable number of Olig2 ${ }^{+}$cells that did not have NG2, indicating a subpopulation of Olig2-expressing cells that are NG2negative in the adult CNS. For example, in the thalamus and midbrain, only $13-16 \%$ of Olig2 ${ }^{+}$cells were $\mathrm{NG}^{+}$ cells (see Table 1 for quantification). What are the majority of the Olig2 ${ }^{+}$but $\mathrm{NG}^{-}$cells in the thalamus and midbrain? What about the other CNS regions in the adult mice? To answer these questions, we further conducted a series of experiments to characterize the Olig2 ${ }^{+}$cells throughout various CNS regions.

\section{Oligodendrocytes are only part of the Olig2-expressing cells}

Oligodendrocytes are myelinating cells that are differentiated from OPCs under the control of bHLH transcriptional factor Olig2 [17]. Besides OPCs, we also analyzed the expression pattern of Olig2 in oligodendrocytes by co-staining Olig2 with two typical markers of oligodendrocytes, CNPase and CC1 [18]. CNPase, comprising about $4 \%$ of the total myelin protein in the CNS, is widely expressed in pre-myelinating and myelinating oligodendrocytes [19]. We detected CNPase expression in the whole brain (Fig. 2a) and spinal cord (Fig. 2n), reflecting a uniform distribution of oligodendrocytes in the adult CNS. At high magnification, we found that all the CNPase-expressing oligodendrocytes had Olig2

(See figure on next page.)

Fig. 1 Co-immunostaining of Olig2 and NG2 in adult mouse CNS. a Sagittal overview of Olig2 and NG2 expression in the mouse brain. b-m High magnification images showing the expression relationship between Olig2 and NG2 in 12 selected brain regions. Note that nearly all NG2 ${ }^{+}$cells are co-stained with Olig2 as indicated by white arrowheads, while a considerable number of Olig2 ${ }^{+}$cells do not have NG2 expression, as indicated by white arrows. In the HIP, about $76.1 \%$ of Olig2 ${ }^{+}$cells had NG2 co-expression, which is the highest among selected regions. $\mathbf{n}$ Coronal overview of the mouse SC, showing the co-immunostaining of Olig2 and NG2. o High magnification image showing the colocalization of Olig2 and NG2 in the $\mathrm{SC}$ of mouse. Note that almost all $\mathrm{NG}^{+}$cells were positive for Olig2 as in other brain regions, but only $8.3 \%$ of Olig2 ${ }^{+}$cells were positive for NG2. Scale bars: $1000 \mu \mathrm{m}(\mathbf{a}), 50 \mu \mathrm{m}(\mathbf{b}-\mathbf{m}, \mathbf{0}), 500 \mu \mathrm{m}(\mathbf{n})$ 

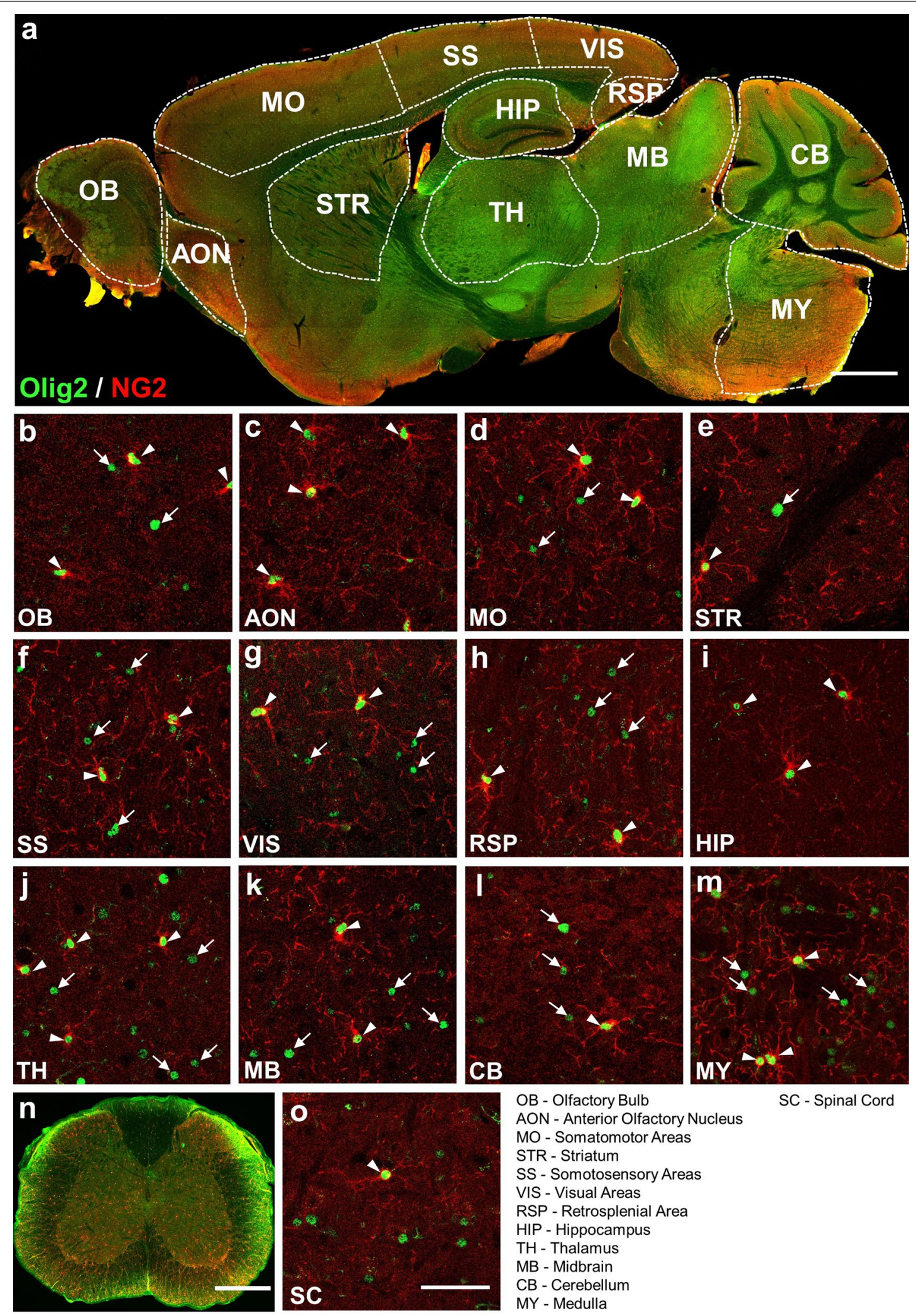

$O B$ - Olfactory Bulb

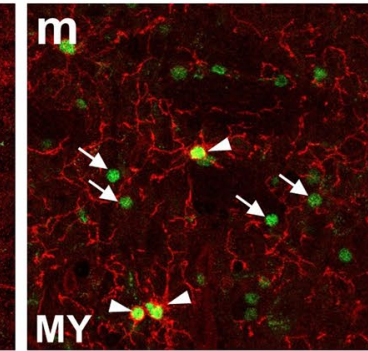

AON - Anterior Olfactory Nucleus

MO - Somatomotor Areas

STR - Striatum

SS - Somotosensory Areas

VIS - Visual Areas

RSP - Retrosplenial Area

HIP - Hippocampus

$\mathrm{TH}$ - Thalamus

MB - Midbrain

$\mathrm{CB}$ - Cerebellum

MY - Medulla 
Table 1 The proportion (\%) of Olig2-expressing cells corresponding to specific glial cell types in different CNS regions

\begin{tabular}{|c|c|c|c|c|}
\hline & \multicolumn{4}{|l|}{ Marker } \\
\hline & $\mathrm{CC}^{+}{ }^{+}$ & CNPase $^{+}$ & $\mathrm{NG2}^{+}$ & $\mathrm{S} 100 \beta^{+}$ \\
\hline \multicolumn{5}{|l|}{ Area } \\
\hline OB & $88.1 \pm 2.5$ & $21.0 \pm 4.3$ & $32.9 \pm 1.4$ & $67.5 \pm 4.8$ \\
\hline AON & $85.1 \pm 2.5$ & $20.4 \pm 1.9$ & $73.7 \pm 8.4$ & $20.1 \pm 1.4$ \\
\hline MO & $94.3 \pm 1.2$ & $43.1 \pm 8.1$ & $17.6 \pm 2.5$ & $13.2 \pm 3.5$ \\
\hline STR & $51.7 \pm 9.1$ & $13.3 \pm 3.6$ & $61.3 \pm 7.9$ & $25.2 \pm 3.5$ \\
\hline SS & $92.2 \pm 2.0$ & $69.1 \pm 4.2$ & $16.7 \pm 1.7$ & $8.5 \pm 2.6$ \\
\hline VIS & $83.0 \pm 2.4$ & $72.9 \pm 2.7$ & $30.7 \pm 1.2$ & $8.8 \pm 0.5$ \\
\hline RSP & $85.1 \pm 1.6$ & $82.8 \pm 1.4$ & $24.3 \pm 1.1$ & $9.6 \pm 3.0$ \\
\hline DG & $66.5 \pm 3.9$ & $12.7 \pm 7.6$ & $76.1 \pm 4.4$ & $2.8 \pm 1.9$ \\
\hline TH & $89.4 \pm 1.5$ & $47.8 \pm 4.8$ & $13.8 \pm 0.9$ & $39.8 \pm 1.7$ \\
\hline$M B$ & $90.9 \pm 1.4$ & $84.3 \pm 1.5$ & $15.6 \pm 2.4$ & $31.6 \pm 0.6$ \\
\hline$C B$ & $97.0 \pm 1.3$ & $92.6 \pm 0.8$ & $13.8 \pm 0.3$ & $6.5 \pm 1.2$ \\
\hline MY & $94.0 \pm 1.4$ & $88.1 \pm 1.2$ & $13.3 \pm 1.7$ & $28.4 \pm 2.5$ \\
\hline SC & $77.0 \pm 1.2$ & $89.4 \pm 1.2$ & $8.3 \pm 0.7$ & $40.6 \pm 2.1$ \\
\hline
\end{tabular}

The proportion of different cell types in Olig2 ${ }^{+}$cells varied according to different CNS regions $(n=4)$. In all regions, more than $50 \%$ Olig2 $^{+}$cells are CC1-positive. However, the proportion of $\mathrm{CNPase}^{+}$and $\mathrm{NG}^{+}$cells had a high variation. As for $\mathrm{S} 100 \beta^{+}$cells, the proportion in most of the selected regions were below $50 \%$ except in the $\mathrm{OB}(67.5 \%)$. Values are presented as mean \pm SEM

immunofluorescence signal (Fig. 2b-m, o). However, not every Olig2 ${ }^{+}$cells showed CNPase signal (Fig. $2 b-m$, o, Table 1), indicating the diversification of the proportion of oligodendrocytes in the adult CNS.

Next, we performed co-immunostaining of Olig2 with mature oligodendrocyte marker CC1. Unlike myelin protein CNPase, CC1 was highly localized in the soma of the oligodendrocytes (Fig. 3). Our data showed that a majority of $\mathrm{CC}^{+}$cells (red) were co-stained with Olig2 (green) throughout the brain $(91.0 \% \sim 99.6 \%$, white arrowheads, Fig. $3 \mathrm{~b}, \mathrm{~d}-\mathrm{m})$ and spinal cord $(98.70 \%$, white arrowheads, Fig. 3n). On the other hand, while most brain regions showed high colocalization between Olig2 and CC1, we also found Olig2 ${ }^{+}$but $\mathrm{CC}^{-}$cells in some CNS regions (Fig. $3 \mathrm{~b}-\mathrm{d}, 3 \mathrm{f}-\mathrm{o}$, and Table 1). Particularly, in the striatum only $51.7 \%$ of Olig2 ${ }^{+}$cells showed CC1 signal, suggesting that many Olig2 ${ }^{+}$cells are not oligodendrocytes in the striatum (Fig. 3e and Table 1). Together, these data suggest that there is a subpopulation of Olig2 ${ }^{+}$cells in some adult CNS regions that are neither oligodendrocytes nor OPCs.

\section{Olig2 not detected in neurons and microglia}

Neurons are electrically excitable cells that are the basic functional units of the CNS. Olig2 plays a crucial role in the generation of motor neurons in the developing spinal cord. We investigated whether Olig2 could be continuously expressed in some neurons of the adult CNS. However, we did not find any $\mathrm{NeuN}^{+}$neurons that coimmunolabeled with Olig2 in either the brain or spinal cord (Fig. 4). Furthermore, we also performed co-immunostaining of Olig2 with microglia marker Iba1 and did not detect any Olig2 ${ }^{+}$cells co-expressing Iba1 (Fig. 5). These data indicate that both neurons and microglia do not express Olig2 in the adult mouse CNS.

\section{Region-specific expression of Olig2 in astrocytes}

Astrocytes are resident glial cells in the CNS besides oligodendrocytes and microglia. We further investigated whether Olig2 expression can be detected in the astrocytes of adult CNS by performing co-immunostaining of Olig2 with S100 $\beta$, an astrocyte specific marker. As shown in the tiled Fig. 6a, S100 $\beta$ signal was found throughout the adult CNS, with very high level immune reactivity detected in the $\mathrm{OB}, \mathrm{AON}, \mathrm{MO}$, and the molecular layer of the cerebellum. Interestingly, high magnification confocal images found that many $\mathrm{S}_{100} \beta^{+}$astrocytes also expressed Olig2 in a region-specific pattern. In the AON, STR, HIP, CB (white arrows, Fig. 6c, e, i, l) and cortical areas including the MO, SS, VIS and the RSP (white arrows, Fig. 6d, f-h), very few Olig2 was located within astrocytes (quantified in Fig. 6p). Unexpectedly, we found a large number of $S 100 \beta^{+}$astrocytes that were also Olig2positive in the $\mathrm{OB}, \mathrm{TH}, \mathrm{MB}$, and $\mathrm{MY}$ (white arrowheads, Fig. 6b, j, k, m). Quantified data showed that the majority of astrocytes $(>70 \%)$ in these regions co-expressed Olig2 (Fig. 6p). An even higher proportion of $\mathrm{S} 100 \beta^{+}$astrocytes co-expressed Olig2 that were detected in the adult mouse spinal cord (89.3\%, Fig. 6n-p). We also calculated the percentage of $\mathrm{S} 100 \beta^{+}$and Olig2 ${ }^{+}$astrocytes among the total Olig $2^{+}$cells, and found that in some areas such

\footnotetext{
(See figure on next page.)

Fig. 2 Co-immunostaining of Olig2 and CNPase in adult mouse CNS. a Sagittal overview of Olig2 and CNPase expression in the mouse brain. b- $\mathbf{m}$ Representative high magnification images illustrating the expression relationship between Olig2 and CNPase in selected brain regions. All $\mathrm{CNPase}^{+}$cells are co-stained with Olig2 as indicated by white arrowheads. On the other hand, Olig2 ${ }^{+}$cells that are not co-stained with CNPase are indicated by white arrows. The proportion of the CNPase and Olig2 double positive cells in total Olig2 ${ }^{+}$cells ranges from $12.7 \%$ to $92.6 \%$ among selected regions. $\mathbf{n}$ Coronal overview of the mouse SC, showing co-immunostaining of Olig2 and CNPase. $\mathbf{o}$ High magnification image showing the co-immunostaining of Olig2 and CNPase in the SC of mouse. Similar to the brain, almost all CNPase ${ }^{+}$cells were positive for Olig2. On the contrary, about $89.4 \%$ of Olig2 ${ }^{+}$cells were co-labelled with CNPase. Scale bars: $1000 \mu \mathrm{m}(\mathbf{a}), 50 \mu \mathrm{m}(\mathbf{b}-\mathbf{m}, \mathbf{0}), 500 \mu \mathrm{m}(\mathbf{n})$
} 

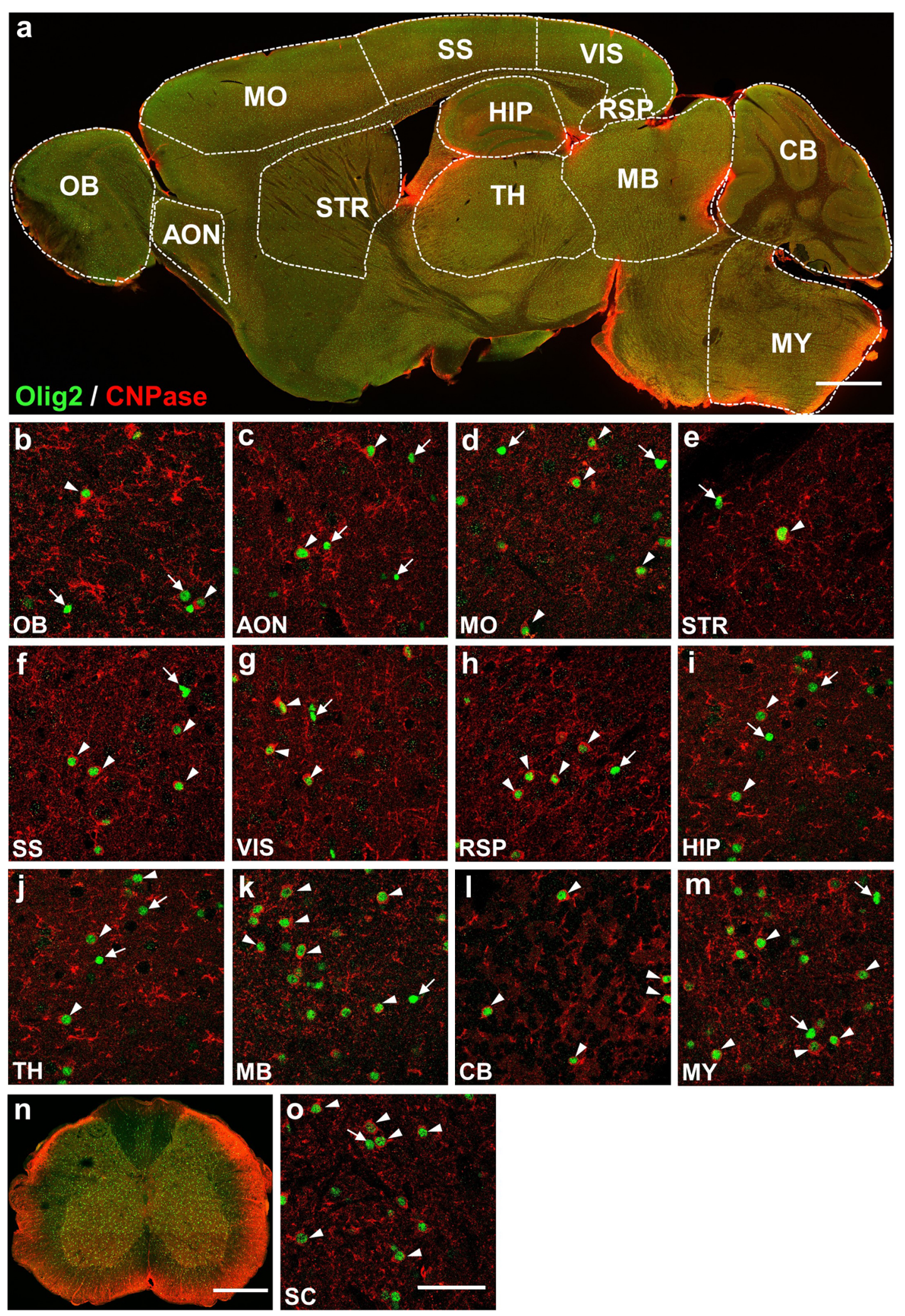
as the $\mathrm{OB}, \mathrm{TH}, \mathrm{MB}, \mathrm{MY}$ and $\mathrm{SC}$, more than a quarter of Olig2 ${ }^{+}$cells are astrocytes (Table 1 ). Therefore, our results suggest that there is a distinct subpopulation of astrocytes with a high level of Olig2 expression in some specific regions in the adult mouse CNS.

To further confirm that astrocytes have Olig2 in some specific CNS regions, we performed co-immunostaining of Olig2 with another astrocytic marker Sox9 [20]. Similarly, we detected a significant number of Sox $9^{+}$cells that were also Olig2-positive in the TH, MB, MY and SC (white arrowheads, Additional file 1: Figure S1j, k, m and o). Quantified data showed that over half of Sox $9^{+}$cells also expressed Olig2 in the TH, MB, MY and SC. Especially, over $90 \%$ of Sox $9^{+}$cells in the spinal cord were also Olig2-expressing cells. Together, our results further confirm that astrocytes can express Olig2 in some regions of adult mouse brain and spinal cord.

\section{Highest Olig2 expression level in OPCs}

After discovering remarkable Olig2 expression in astrocytes within some specific regions, we further investigated whether the Olig2 expression level in astrocytes is comparable to that of OPCs and oligodendrocytes. When compared side-by-side among astrocytes, OPCs, and oligodendrocytes, we found that OPCs expressed much higher level of Olig2 signal than that of oligodendrocytes and astrocytes in the $\mathrm{OB}$ (Fig. $7 \mathrm{a}-\mathrm{c}$ ), and $\mathrm{TH}, \mathrm{MB}$, and MY, as well as the spinal cord (Fig. $7 d, p<0.001$ ). Interestingly, the Olig2 expression level was comparable between oligodendrocytes and astrocytes (Fig. 7). These results indicate that in some regions of the adult mouse CNS under normal conditions, astrocytes can express Olig2 at a similar level to the oligodendrocytes.

\section{Discussion}

In this study, we discovered that Olig2 was expressed not only in OPCs and oligodendrocytes but also in some astrocytes in the adult mouse CNS. Most intriguingly, the Olig2-expressing astrocytes showed clear regional specificity with high enrichment in certain regions such as OB, STR, TH, MB, MY, and spinal cord. Such distinct subpopulation of Olig2 ${ }^{+}$astrocytes underscores the heterogeneity of astrocytes and implies potential functional divergence from Olig2 ${ }^{-}$astrocytes.

\section{Astrocyte origination and heterogeneity}

During gliogenesis, both astrocytes and oligodendrocytes originate from radial glial cells that are derived from neuroepithelial cells [3,21]. Different subtypes of astrocytes may be dependent on the differential combination of SLIT1 and Reelin under the regulation of different transcription factors activated by SHH signaling [3]. Interestingly, in the mouse spinal cord, a subpopulation of Olig2 ${ }^{+}$astrocyte progenitor cells are reported to be produced in the same region where oligodendrocytes are generated $[7,10]$, which might explain why we observed a large number of Olig2 ${ }^{+}$astrocytes in the adult spinal cord. Besides produced directly from the transformation of radial glia cells, astrocytes can also be produced from the proliferation of differentiated astrocytes [3, 22, 23]. Therefore, both embryonic and postnatal astrogliosis contribute to the complex origination of astrocytes. It will be interesting to trace back where the Olig2 ${ }^{+}$astrocytes are generated: the embryonic radial glia or postnatal astrocytes.

Astrocytes are ubiquitous in the adult CNS, playing key roles in structural and trophic support, including bloodbrain-barrier formation, ion homeostasis, and synaptic transmission [24]. Ramón y Cajal's seminal work identified two classic groups of astrocytes: fibrous astrocytes in the white matter and protoplasmic astrocytes in the gray matter. Recent studies demonstrate that astrocytes may have region-specific characteristics in morphology [25], gene expression [26] and function [27]. Our study here discovers that Olig2, an oligodendroglia-specific marker, is highly expressed in certain astrocytes within some discrete CNS regions, suggesting that Olig2 might be an important regulator of astrocyte heterogeneity. Previously, Xin et al. [28] reported that glutamine synthetase (GS), an astrocyte-specific marker, is highly expressed in oligodendrocytes in the midbrain and spinal cord. Combined with our finding of a region-dependent Olig2 expression in astrocytes, these results together further suggest that astrocytes and oligodendrocytes may share certain common properties, at least in certain regions. In our present study, we found that the Olig2 expression level in astrocytes is comparable to that in CC1-labeled mature oligodendrocytes, but not at the level of OPCs. Future studies may help us to better understand the

\footnotetext{
(See figure on next page.)

Fig. 3 Co-immunostaining of Olig2 and CC1 in adult mouse CNS. a Sagittal overview of Olig2 and CC1 expression pattern in the mouse brain. b- $\mathbf{m}$ High magnification images showing the expression relationship between Olig2 and CC1 in brain regions of interest. Most of the CC ${ }^{+}$cells have Olig2 immuno signal as indicated by white arrowheads. On the other hand, white arrows indicate Olig2 ${ }^{+}$cells without CC1. The proportion of $\mathrm{CC}^{+}$and Olig2 $2^{+}$cells is about $50 \%$ in the STR and HIP, and higher than $80 \%$ in other brain regions. $\mathbf{n}$ Coronal overview of the mouse SC, showing co-immunostaining of Olig2 and CC1. o High magnification image displaying the co-immunostaining of Olig2 and CC1 in the SCe. As in the brain regions, the majority of the $\mathrm{CC}^{+}$cells are positive for Olig2 (white arrowheads), quantified data showing about $77.0 \%$ of $\mathrm{Olig}^{+} \mathrm{Cells}^{\mathrm{are}} \mathrm{CC} 1^{+}$ (white arrows). Scale bars: $1000 \mu \mathrm{m}(\mathbf{a}), 50 \mu \mathrm{m}(\mathbf{b}-\mathbf{m}, \mathbf{0}), 500 \mu \mathrm{m}(\mathbf{n})$
} 

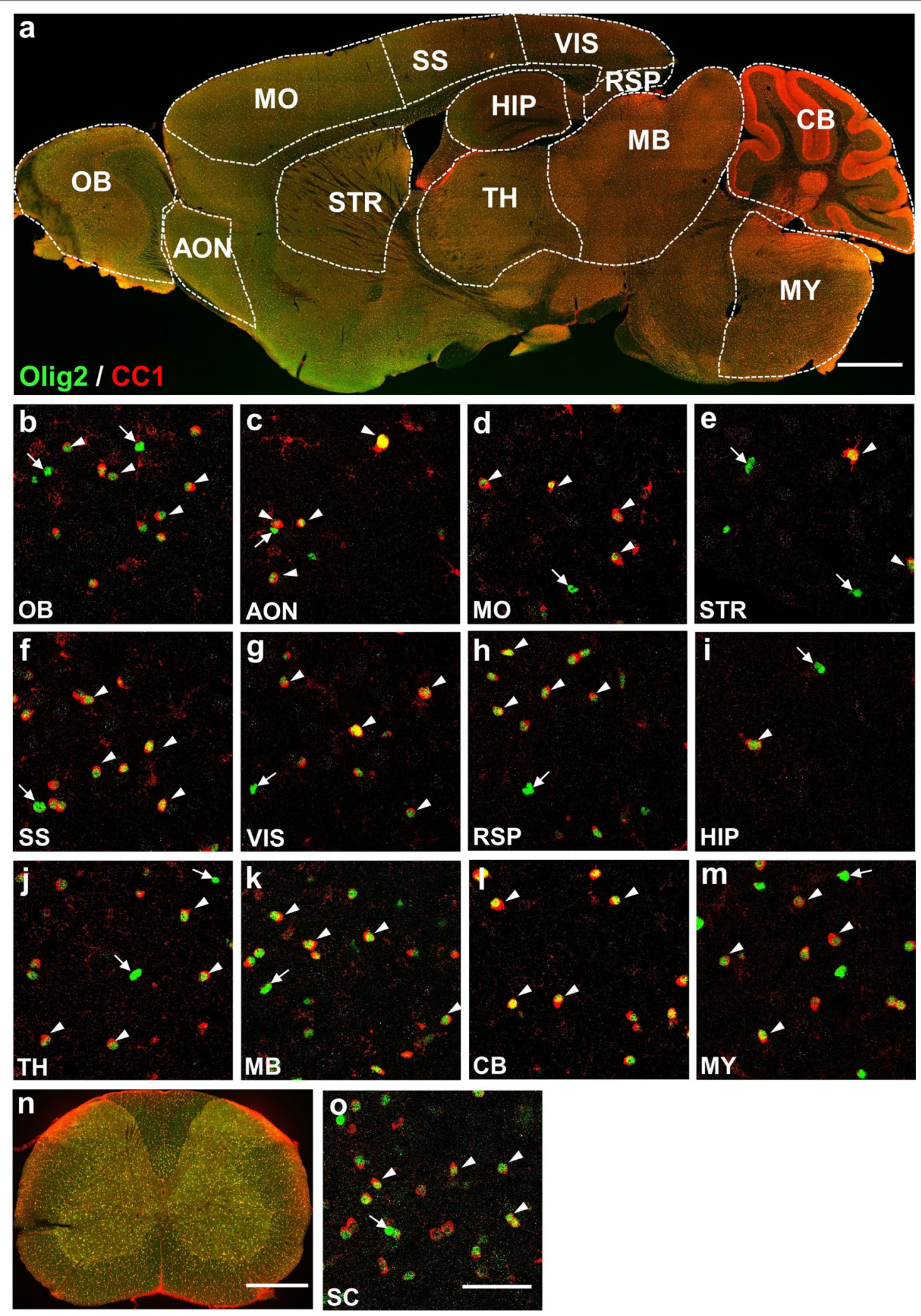
relationship between Olig2 ${ }^{+}$astrocytes and $\mathrm{GS}^{+}$oligodendrocytes in the adult CNS.

\section{Olig2 and astrocyte fate}

Olig2 has been reported to play a critical role not only in the specification and differentiation of oligodendroglia, but also in regulating astrocytic fate during early neural development. Lineage tracing studies found that a subset of Olig2 ${ }^{+}$progenitor cells can differentiate into astrocytes, accompanied by downregulation of Olig2 [7, 29]. In neuroepithelial cells, knockout of Olig2 resulted in the differentiation of astrocytes but not oligodendrocytes [30], whereas overexpression of Olig2 inhibited the generation of astrocytes [31]. In vivo studies in prenatal and postnatal mice have also found that forced inhibition of Olig2 is sufficient for the fate switch from oligodendroglia to astrocytes [29], suggesting that Olig2 may function as a switch to turn glial fate toward astrocytes or oligodendrocytes. Interestingly, a subpopulation of $\mathrm{S} 100 \beta^{+}$astrocytes have been clearly labeled in Olig2-transgenic mice [32]. Conversely, a subpopulation of oligodendrocytes and OPCs are also clearly labeled in S100 $\beta$-transgenic mice $[33,34]$. Our studies reveal that there are widespread Olig $2^{+}$astrocytes throughout the brain and spinal cord, and in certain regions the ratio of Olig2 ${ }^{+}$astrocytes can be very high such as that in the $\mathrm{OB}$ with $67.5 \%$ being Olig2 ${ }^{+}$astrocytes. Therefore, while Olig2 is a key factor favoring the fate-determination of oligodendroglia, some glial cells appear to adopt astrocytic fate regardless the presence of Olig2.

\section{Olig2 and astrocyte function}

Under brain injury and disease conditions such as Alzheimer's disease and ischemic stroke, Olig2 is robustly upregulated in reactive astrocytes $[14,15,35]$. On the other hand, Olig2 ablation in astrocytes dramatically reduces the formation of reactive astrocytes as well as their proliferation [15] while improving endogenous neurogenesis $[35,36]$. Since reactive astrocytes play a protective role in response to injury, the upregulation of Olig2 in reactive astrocytes may be involved in the mechanism of early CNS self-defense in adult animals. However, in later stage of injury or disease, Olig $2^{+}$reactive astrocytes may be detrimental to neural recovery. Our current finding of Olig2 ${ }^{+}$astrocytes in the resting state of adult CNS certainly raises an important question of what is the function of Olig2 in these differentiated adult astrocytes? Since Olig2 is a bHLH transcription factor, it can form dimer with other bHLH factors including neural transcription factors such as NeuroD1 and neurogenin-2 (Ngn2). Therefore, one speculation of the Olig2 function in the adult astrocytes is an inhibitory function to prevent neurogenesis due to accidental activation of neural transcription factors. Another possibility is that since Olig2 can directly bind to chromosomal DNA and regulate the expression of many downstream genes, including both neuronal and glial genes, the presence of Olig2 in the adult astrocytes is an assurance that neuronal genes will be constantly inhibited. Another hypothesis is that these Olig ${ }^{+}$astrocytes are more sensitive to environmental changes, such as injury and disease, and therefore will respond rapidly to various stimulations in order to provide adequate protections to the surrounding cells. It will be important for future studies to dissect out the precise function of these Olig2 ${ }^{+}$astrocytes in different disease states such as Alzheimer's disease and stroke.

Here, we have identified a large population of mature astrocyte that maintain expression of Olig2 in the adult mouse CNS, this observation raises the question of whether the expression of Olig2 confers specific functional properties to Olig2 expression astrocytes, that needs further study in the future.

\section{Conclusion}

This study presents a comprehensive Olig2 expression pattern in the adult mouse CNS, and identifies a subtype of Olig2 ${ }^{+}$astrocytes that are regionally enriched in the brain and spinal cord.

\section{Methods \\ Animals}

Wild-type, 5-month-old C57BL/6 mice were used for most of the experiments and analysis. Both male $(\mathrm{n}=2)$ and female mice $(n=2)$ were used in this study. The mice were housed in a 12/12-h light-dark cycle room and received food and water ad libitum. Experimental

\footnotetext{
(See figure on next page.)

Fig. 4 Co-immunostaining of Olig2 and NeuN in adult mouse CNS. a Sagittal overview of Olig2 and NeuN expression pattern in the mouse brain. b-m High magnification images showing the expression relationship between Olig2 and NeuN in selected brain regions. Co-localization of Olig2 and NeuN is not observed in all the regions of interest as indicated by white arrows. $\mathbf{n}$ Coronal overview of the mouse SC. $\mathbf{o}$ A high magnification image depicting the co-immunostaining of Olig2 and NeuN in the SC. Similarly, no Olig2 is expressed in $\mathrm{NeuN}^{+}$cells. White arrows indicate Olig2 ${ }^{+}$ cells without NeuN co-localization. Scale bars: $1000 \mu \mathrm{m}(\mathbf{a}), 50 \mu \mathrm{m}(\mathbf{b}-\mathbf{m}, \mathbf{0}), 500 \mu \mathrm{m}(\mathbf{n})$
} 

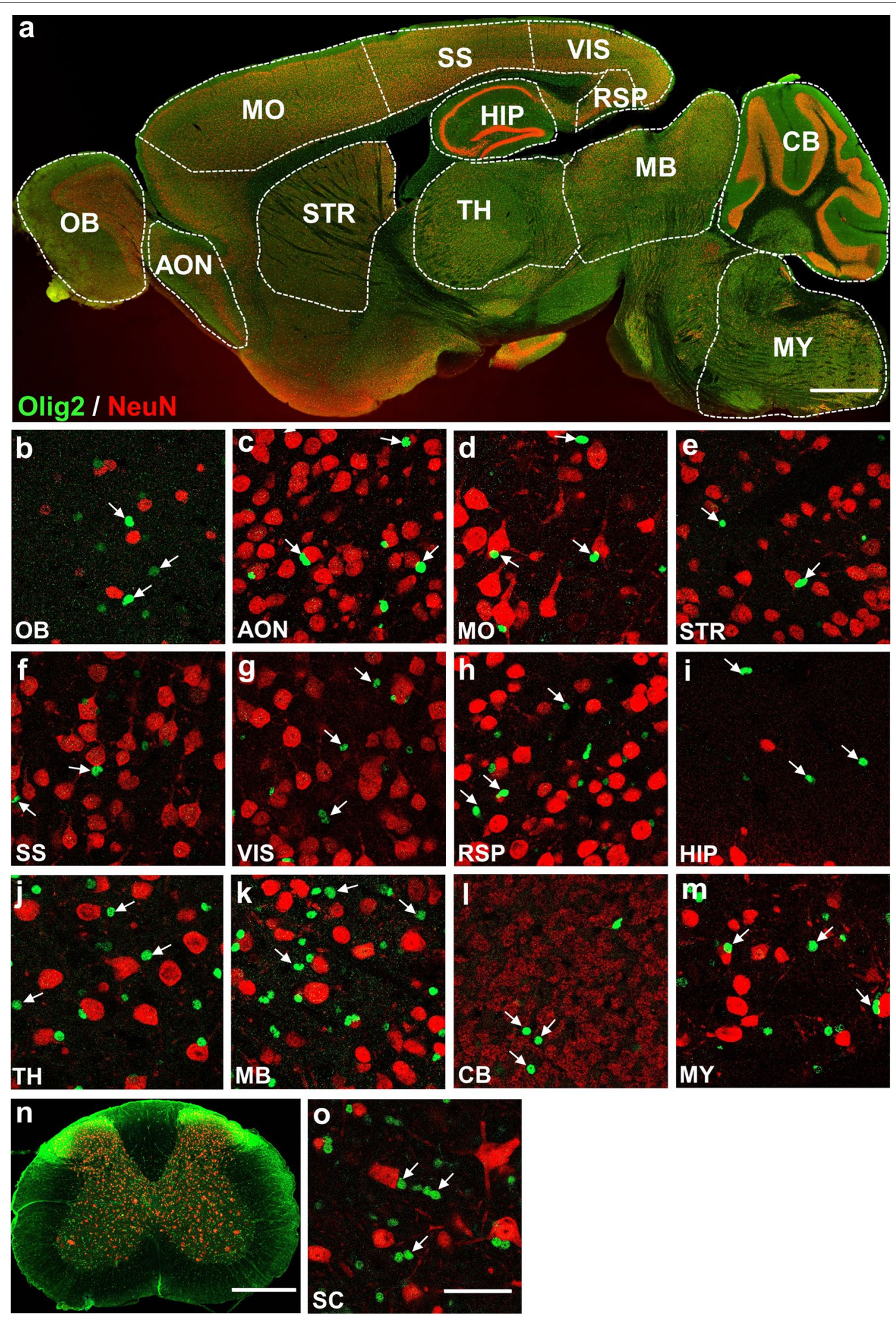
Wang et al. Mol Brain $\quad$ (2021) 14:36

Page 10 of 14

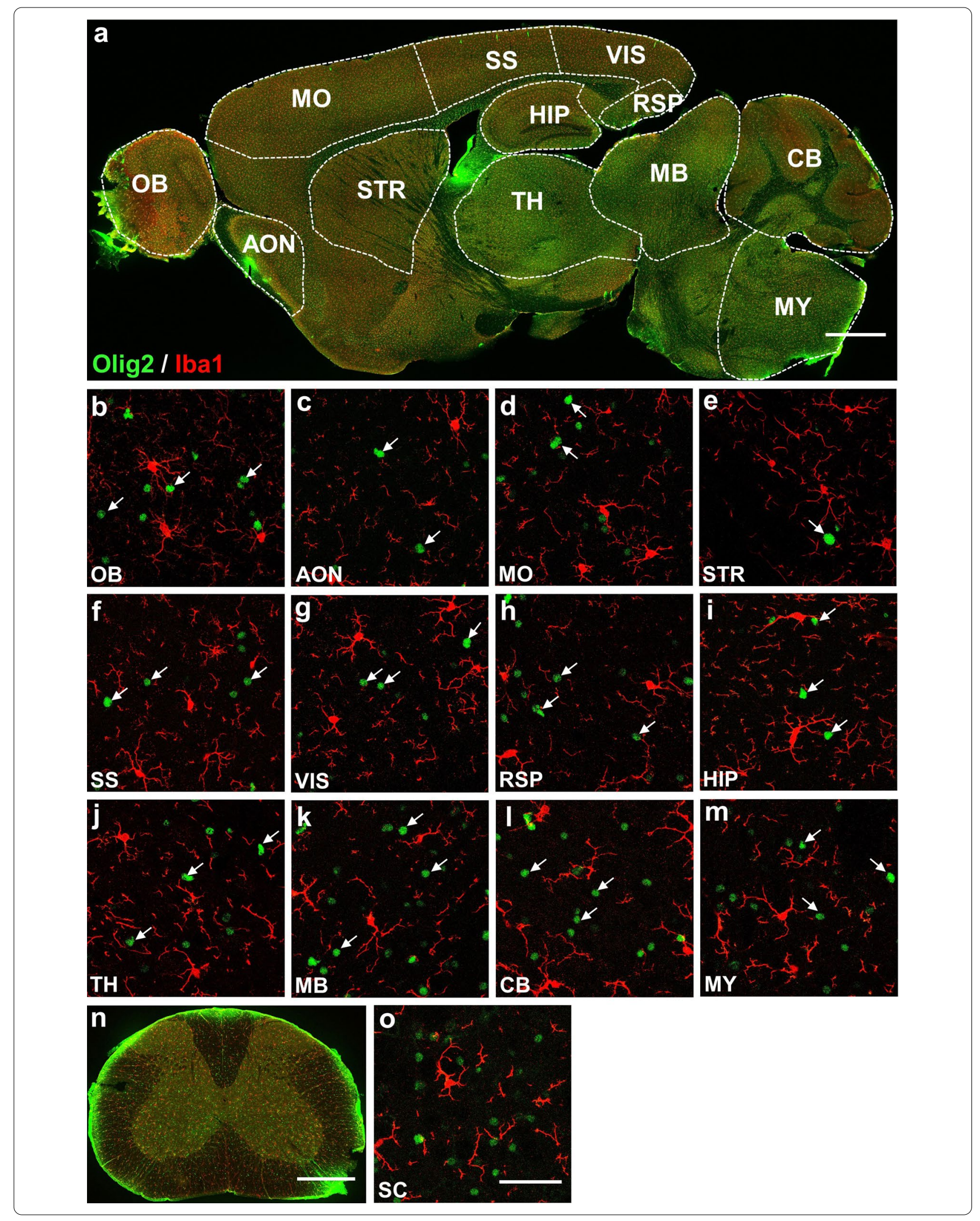


(See figure on previous page.)

Fig. 5 Co-immunostaining of Olig2 and Iba1 in adult mouse CNS. a Sagittal overview of Olig2 and Iba1 expression in the mouse brain. b-m High magnification images showing the expression relationship between Olig2 and Iba1 in selected brain regions. There is no co-localization of Olig2 and Iba1 in all the regions of interest as indicated by white arrows. $\mathbf{n}$ Coronal overview of the mouse SC. $\mathbf{o}$ A high magnification image presenting there is no co-immunostaining of Olig2 and Iba1 in the SC (white arrows). Scale bars: $1000 \mu \mathrm{m}(\mathbf{a}), 50 \mu \mathrm{m}(\mathbf{b}-\mathbf{m}, \mathbf{0}), 500 \mu \mathrm{m}(\mathbf{n})$

protocols were approved by the Pennsylvania State University IACUC, and procedures were performed in line with the guidelines of the National Institute of Health (NIH).

For Sox9 and Olig2 immunostaining study, the 3-month-old C57BL/6 mice were used for the analysis (total 4 mice, 2 male and 2 female). The mice were housed in a $12 \mathrm{~h}$ light/dark cycle and supplied with enough food and water. Experimental protocols were approved by Jinan University IACUC.

\section{Immunohistochemistry and analysis}

Mice were anesthetized with Avertin (tribromoethanol, $250 \mathrm{mg} / \mathrm{kg}$, i.p.) and then quickly transcardially perfused with ice-cold artificial cerebrospinal fluid (aCSF). The brain and spinal cord were removed and post-fixed in $4 \%$ PFA overnight in darkness at $4{ }^{\circ} \mathrm{C}$. The spinal cord was then dehydrated in 30\% sucrose for another day. The brain sample was sagittally sectioned at $40 \mu \mathrm{m}$ with a vibratome (Leica, VTS1000), and the spinal cord was coronally sectioned at $40 \mu \mathrm{m}$ with a cryostat (Leica, CM1950), then slices were stored in $0.1 \mathrm{M} \mathrm{PB}$ at $4{ }^{\circ} \mathrm{C}$. Both brain and spinal cord slices were rinsed three times with PBS for ten minutes per rinse. Slices were blocked with $0.3 \%$ triton $\mathrm{PBS}+5 \%$ normal donkey serum (NDS) for two hours at room temperature. Slices were incubated with primary antibodies diluted in $0.05 \%$ triton PBS $+5 \%$ NDS in a moist environment for two nights in darkness at $4^{\circ} \mathrm{C}$. Then slices were rinsed three times with PBS for ten minutes per rinse and incubated with secondary antibodies diluted in $0.05 \%$ triton PBS + $5 \%$ NDS for two hours at room temperature. After three times of washing using PBS, the slices were mounted on glass slides with anti-fading solution. For double immunostaining, slices were simultaneously incubated with Olig2 respectively with S100ß, CC1, CNPase, NG2, NeuN, or Iba1. The following primary antibodies were used for immunohistochemistry: rabbit anti-Olig2 (Millipore, 1:1000), mouse anti-S100 $\beta$ (Abcam, 1:1000), mouse anti-CC1 (Abcam, 1:300), mouse anti-CNPase (Abcam, 1:1000), mouse antiOlig2 (Millipore, 1:1000), rabbit anti-NG2 (Millipore, 1:200), guinea pig anti-NeuN (Millipore, 1:2000), rabbit anti-Iba1 (Wako, 1:1000). All secondary antibodies were Alexa Fluor-conjugated from Jackson ImmunoResearch that included donkey anti-rabbit 488 , donkey anti-mouse 594, donkey anti-mouse 488, donkey anti-rabbit-594, donkey anti-guinea pig 647, donkey anti-rabbit 594.

The images were acquired by Olympus FV1200 and Zeiss confocal microscope (LSM 800). For quantification in each CNS region of interest, at least 4 areas of $40 \times$ lens were randomly selected and imaged. The white matter cells of mouse CNS were not involved in this study. The quantification of Olig2 intensity was completed by the Zeiss software ZEN. The intensity indicates the mean gray value: mean gray value $=$ integrated signal density/ area.

\section{Statistical analysis}

The comparison of Olig2 fluorescence intensity among groups was analyzed by One-Way ANOVA test. Data were presented as mean \pm standard mean of error (SEM). GraphPad Prism software was used for data analysis and

\footnotetext{
(See figure on next page.)

Fig. 6 The percentage of Olig2 ${ }^{+}$astrocytes in different mouse CNS regions. a Sagittal overview of the mouse brain indicating a variety of brain regions of interest. $\mathbf{b}-\mathbf{m}$ High magnification images showing the olig2 and $5100 \beta$ double immune-staining in different mouse brain regions. White arrowheads indicate co-localization of Olig2 and $\mathrm{S100 \beta}$, while white arrows indicate astrocytes that do not express Olig2. Note that in the AON (c), STR (e), HIP (i), CB (I) and the cortical areas including the MO (d), SS (f), VIS $(\mathbf{g})$ and the RSP (h), few astrocytes expresse Olig2, while in the OB $(\mathbf{b}), \mathrm{TH}(\mathbf{j}), \mathrm{MB}(\mathbf{k})$, and the MY (m), majority astrocytes (> 70\%) have Olig2 immune reactivity. $\mathbf{n}$ Coronal overview of the mouse SC showing Olig2 ${ }^{+}$ astrocytes. $\mathbf{o}$ High magnification image displaying the co-localization of Olig2 and S100 $\beta$ in the SC. Note that most of the astrocytes (89.3\%) in the SC expresse Olig2. $\mathbf{p}$ The ratio of Olig2 ${ }^{+}$cells in total of the $\mathrm{S} 100 \beta^{+}$cells in each brain region and the SC. Data are represented as mean \pm SEM. Scale bars: $1000 \mu \mathrm{m}(\mathbf{a}), 50 \mu \mathrm{m}(\mathbf{b}-\mathbf{m}, \mathbf{0}), 500 \mu \mathrm{m}(\mathbf{n})$
} 

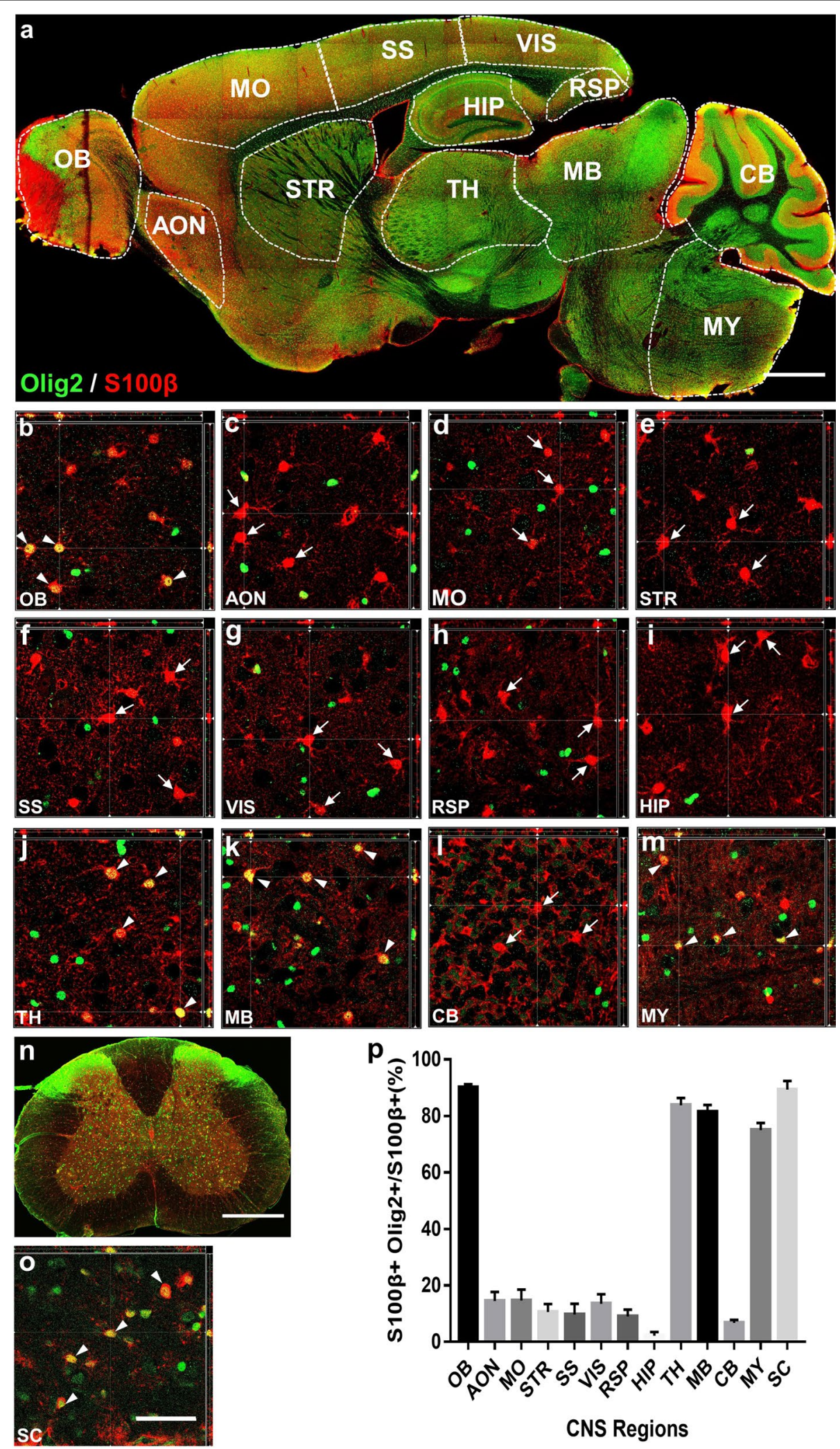

CNS Regions 

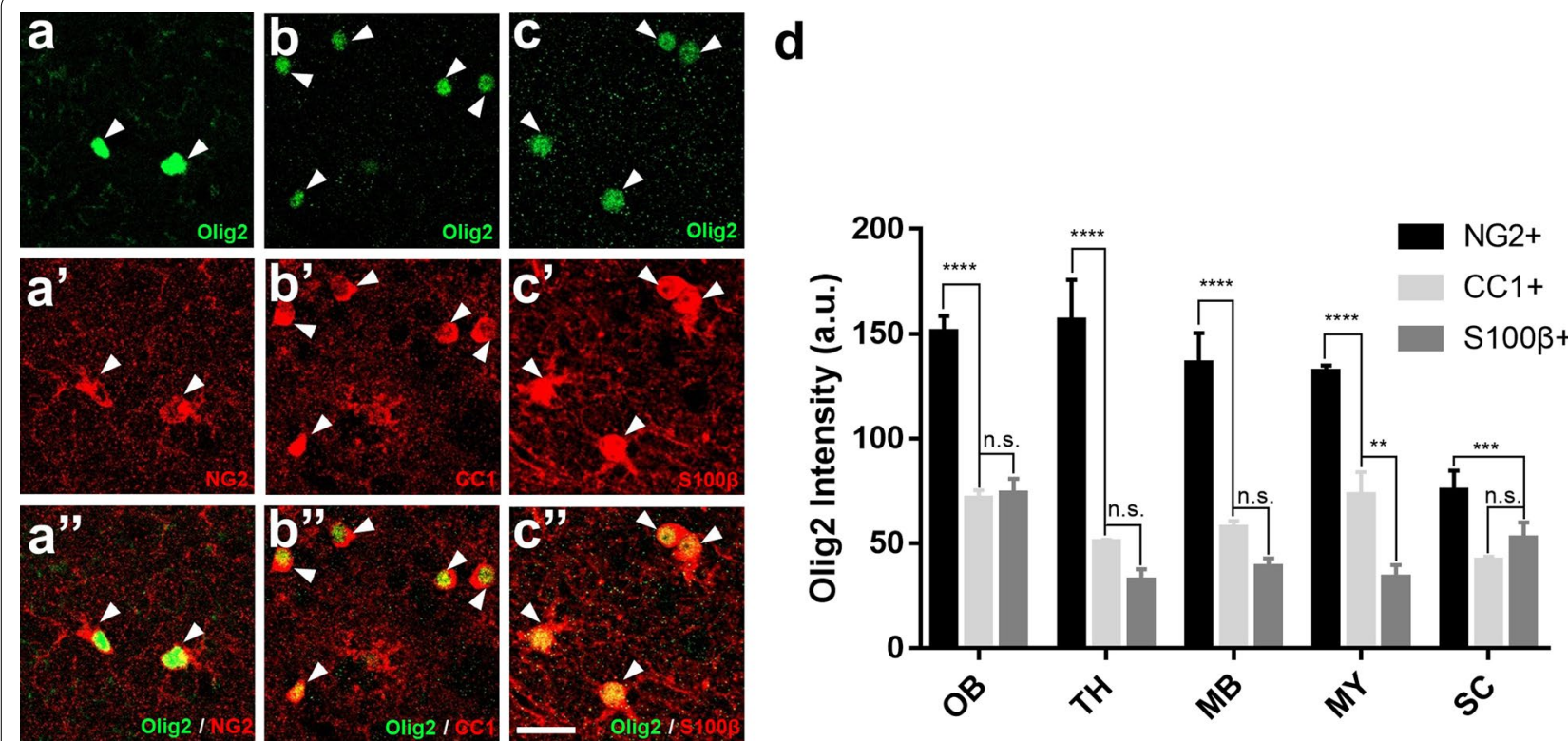

Fig. 7 Comparison of Olig2 fluorescence intensity in OPCs, oligodendrocytes and astrocytes. $\mathbf{a}-\mathbf{c}, \mathbf{a}^{\prime}-\mathbf{c}^{\prime}, \mathbf{a}^{\prime \prime}-\mathbf{c}^{\prime \prime}$ Taking the olfactory bulb as an example, the Olig2 signal intensity in $\mathrm{NG}_{2}{ }^{+} \mathrm{OPCs}$ is much stronger than that in $\mathrm{CC}_{1}{ }^{+}$oligodendrocytes and $\mathrm{S}_{100} \beta^{+}$astrocytes. However, there is no significant difference of Olig2 intensity between oligodendrocytes and astrocytes. $\mathbf{d}$ Comprehensive quantitative comparison of fluorescence intensity of Olig2 in OPCs, oligodendrocytes, and astrocytes in the five CNS regions where Olig2 ${ }^{+}$astrocytes are enriched. OPCs has the strongest Olig2 signal. In TH, MB, and MY, there is no significant difference of olig2 signal between oligodendrocytes and astrocytes, but it has significant difference in MY region. In SC, no significant difference was detected between oligodendrocytes and astrocytes. Scale bars: $20 \mu \mathrm{m}$ (a-c, a'-c', $\left.\mathbf{a}^{\prime \prime}-\mathbf{c}^{\prime \prime}\right)$. All values are presented as mean \pm SEM; One-way ANOVA test; ${ }^{* *} p<0.01,{ }^{* * *} p<0.001,{ }^{* * *} p<0.0001$

figure generation. Significance was set at the threshold: " $\mathrm{p}<0.05 ;{ }^{* * \mathrm{p}}<0.01$; **** $\mathrm{p}<0.001$, ***** $\mathrm{p}<0.0001$.

\section{Supplementary Information}

The online version contains supplementary material available at https://doi. org/10.1186/s13041-021-00747-0.

Additional file 1: Table S1. Zeiss Confocal imaging acquisition parameters. Table S2. The proportion (\%) of S100 $\beta^{+}$and Sox9 ${ }^{+}$cells with Olig2 signal in different CNS regions. Figure $\mathrm{S1}$. The percentage of $\mathrm{SOX}^{+}$cells co-labeled with Olig2 in different adult mouse CNS regions.

\section{Abbreviations}

CNS: Central nervous system; OPCs: Oligodendrocyte precursor cells; Olig2: Oligodendrocyte transcription factor 2; bHLH: Basic helix-loop-helix; SC: Spinal cord; TH: Thalamus; MB: Midbrain; OB: Olfactory bulb; AON: Anterior olfactory nucleus; MO: Motor cortex; STR: Striatum; SS: Somatosensory cortex; VIS: Visual cortex; RSP: Retrosplenial area; Hip: Hippocampus; CB: Cerebellum; MY: Medulla; GS: Glutamine synthetase; NIH: National Institute of Health; aCSF: Artificial cerebrospinal fluid; NDS: Normal donkey serum; SEM: Standard mean of error.

\section{Acknowledgements}

We thank Yuting Bai for mouse weaning. We would like to thank Chen Lab members for helpful discussions.

\section{Authors' contributions}

$\mathrm{HW}$ performed the majority of the experiments, analyzed the data and wrote the manuscript. ZW analyzed the data and wrote the manuscript. LX, CYL, KYH, $J L C$, YWG performed the Sox 9 experiments and collected the data, analyzed the data, and made the figure. AS and SS revised the manuscript. CX, ZW, and
GC designed the experiments, supervised the project and revised the manuscript. All authors read and approved the final manuscript.

\section{Funding}

This work was supported by Charles H. "Skip"Smith Endowment Fund at Pennsylvania State University and startup fund at Jinan University to G.C.

\section{Availability of data and materials}

The datasets used and/or analysed during the current study are available from the corresponding author on reasonable request.

\section{Ethics approval and consent to participate}

All of the data in this manuscript was collected from the mouse that is approved by the Pennsylvania State University IACUC and in accordance with guidelines of National Institutes of Health. There is no data that collected from human subjects in this study.

\section{Consent for publication}

Not applicable.

\section{Competing interests}

All authors declare no conflict of interest.

\section{Author details}

${ }^{1}$ Department of Neurology, Affiliated ZhongDa Hospital, School of Medicine, Southeast University, Nanjing 210009, Jiangsu, China. ${ }^{2}$ Department of Biology, Huck Institutes of Life Sciences, Pennsylvania State University, University Park, PA 16802, USA. ${ }^{3}$ GHM Institute of CNS Regeneration, Jinan University, Guangzhou 510632, China. ${ }^{4}$ Institute of Neuropsychiatry, Affiliated ZhongDa Hospital, Southeast University, Nanjing 210009, Jiangsu, China.

Received: 12 September 2020 Accepted: 4 February 2021

Published online: 17 February 2021 


\section{References}

1. Takebayashi H, Yoshida S, Sugimori M, Kosako H, Kominami R, Nakafuku M, Nabeshima Y. Dynamic expression of basic helix-loop-helix Olig family members: implication of Olig2 in neuron and oligodendrocyte differentiation and identification of a new member, Olig3. Mech Dev. 2000;99(1-2):143-8

2. Meijer DH, Kane MF, Mehta S, Liu H, Harrington E, Taylor CM, Stiles CD, Rowitch DH. Separated at birth? The functional and molecular divergence of OLIG1 and OLIG2. Nat Rev Neurosci. 2012;13(12):819-31.

3. Rowitch DH, Kriegstein AR. Developmental genetics of vertebrate glialcell specification. Nature. 2010;468(7321):214-22.

4. Li H, de Faria JP, Andrew P, Nitarska J, Richardson WD. Phosphorylation regulates OLIG2 cofactor choice and the motor neuron-oligodendrocyte fate switch. Neuron. 2011:69(5):918-29.

5. Ravanelli AM, Appel B. Motor neurons and oligodendrocytes arise from distinct cell lineages by progenitor recruitment. Genes Dev. 2015;29(23):2504-15.

6. Zannino DA, Appel B. Olig2+ precursors produce abducens motor neurons and oligodendrocytes in the zebrafish hindbrain. J Neurosci. 2009:29(8):2322-33.

7. Masahira N, Takebayashi H, Ono K, Watanabe K, Ding L, Furusho M, Ogawa Y, Nabeshima Y, Alvarez-Buylla A, Shimizu K, et al. Olig2-positive progenitors in the embryonic spinal cord give rise not only to motoneurons and oligodendrocytes, but also to a subset of astrocytes and ependymal cells. Dev Biol. 2006;293(2):358-69.

8. Dimou L, Simon C, Kirchhoff F, Takebayashi H, Gotz M. Progeny of Olig2expressing progenitors in the gray and white matter of the adult mouse cerebral cortex. J Neurosci. 2008;28(41):10434-42.

9. Tatsumi K, Okuda H, Morita-Takemura S, Tanaka T, Isonishi A, Shinjo T, Terada Y, Wanaka A. Voluntary exercise induces astrocytic structural plasticity in the globus pallidus. Front Cell Neurosci. 2016:10:165.

10. Ohayon D, Escalas N, Cochard P, Glise B, Danesin C, Soula C. Sulfatase 2 promotes generation of a spinal cord astrocyte subtype that stands out through the expression of Olig2. Glia. 2019;67(8):1478-95.

11. Bergles DE, Richardson WD. Oligodendrocyte development and plasticity. Cold Spring Harb Perspect Biol. 2015;8(2):a020453.

12. Yang Y, Vidensky S, Jin L, Jie C, Lorenzini I, Frankl M, Rothstein JD. Molecular comparison of GLT1 + and ALDH1L1+ astrocytes in vivo in astroglial reporter mice. Glia. 2011;59(2):200-7.

13. Zuo H, Wood WM, Sherafat A, Hill RA, Lu QR, Nishiyama A. Agedependent decline in fate switch from NG2 cells to astrocytes after Olig2 deletion. J Neurosci. 2018;38(9):2359-71.

14. Marumo T, Takagi Y, Muraki K, Hashimoto N, Miyamoto S, Tanigaki K. Notch signaling regulates nucleocytoplasmic Olig2 translocation in reactive astrocytes differentiation after ischemic stroke. Neurosci Res. 2013;75(3):204-9.

15. Chen Y, Miles DK, Hoang T, Shi J, Hurlock E, Kernie SG, Lu QR. The basic helix-loop-helix transcription factor olig2 is critical for reactive astrocyte proliferation after cortical injury. J Neurosci. 2008;28(43):10983-9.

16. Guo F, Maeda Y, Ma J, Xu J, Horiuchi M, Miers L, Vaccarino F, Pleasure D. Pyramidal neurons are generated from oligodendroglial progenitor cells in adult piriform cortex. J Neurosci. 2010;30(36):12036.

17. Liu Z, Hu X, Cai J, Liu B, Peng X, Wegner M, Qiu M. Induction of oligodendrocyte differentiation by Olig2 and Sox10: evidence for reciprocal interactions and dosage-dependent mechanisms. Dev Biol. 2007;302(2):683-93.

18. Valerio-Gomes B, Guimaraes DM, Szczupak D, Lent R. The absolute number of oligodendrocytes in the adult mouse brain. Front Neuroanat. 2018;12:90.

19. Verrier JD, Jackson TC, Gillespie DG, Janesko-Feldman K, Bansal R, Goebbels S, Nave KA, Kochanek PM, Jackson EK. Role of CNPase in the oligodendrocytic extracellular 2',3'-cAMP-adenosine pathway. Glia. 2013;61(10):1595-606.
20. Sun W, Cornwell A, Li J, Peng S, Osorio MJ, Aalling N, Wang S, Benraiss A, Lou N, Goldman SA, et al. SOX9 is an astrocyte-specific nuclear marker in the adult brain outside the neurogenic regions. J Neurosci. 2017;37(17):4493-507.

21. Rowitch DH. Glial specification in the vertebrate neural tube. Nat Rev Neurosci. 2004;5(5):409-19

22. Molofsky AV, Deneen B. Astrocyte development: a guide for the perplexed. Glia. 2015;63(8):1320-9.

23. Schitine C, Nogaroli L, Costa MR, Hedin-Pereira C. Astrocyte heterogeneity in the brain: from development to disease. Front Cell Neurosci. 2015;9:76.

24. Verkhratsky A, Nedergaard M. Physiology of astroglia. Physiol Rev. 2018;98(1):239-389.

25. Emsley JG, Macklis JD. Astroglial heterogeneity closely reflects the neuronal-defined anatomy of the adult murine CNS. Neuron Glia Biol. 2006;2(3):175-86.

26. Lozzi B, Huang TW, Sardar D, Huang AY, Deneen B. Regionally distinct astrocytes display unique transcription factor profiles in the adult brain. Front Neurosci. 2020;14:61.

27. Khakh BS, Sofroniew MV. Diversity of astrocyte functions and phenotypes in neural circuits. Nat Neurosci. 2015;18(7):942-52.

28. Xin W, Mironova YA, Shen H, Marino RAM, Waisman A, Lamers WH, Bergles DE, Bonci A. Oligodendrocytes support neuronal glutamatergic transmission via expression of glutamine synthetase. Cell Rep. 2019;27(8):22622271.e2265.

29. Zhu X, Zuo H, Maher BJ, Serwanski DR, LoTurco JJ, Lu QR, Nishiyama A. Olig2-dependent developmental fate switch of NG2 cells. Development. 2012;139(13):2299-307.

30. Takebayashi H, Nabeshima Y, Yoshida S, Chisaka O, Ikenaka K, Nabeshima $Y$. The basic helix-loop-helix factor olig2 is essential for the development of motoneuron and oligodendrocyte lineages. Curr Biol. 2002;12(13):1157-63.

31. Fukuda S, Kondo T, Takebayashi H, Taga T. Negative regulatory effect of an oligodendrocytic bHLH factor OLIG2 on the astrocytic differentiation pathway. Cell Death Differ. 2004;11(2):196-202.

32. Tatsumi $K$, Isonishi A, Yamasaki M, Kawabe Y, Morita-Takemura S, Nakahara K, Terada Y, Shinjo T, Okuda H, Tanaka T, et al. Olig2-lineage astrocytes: a distinct subtype of astrocytes that differs from GFAP astrocytes. Front Neuroanat. 2018;12:8.

33. Vives $V$, Alonso G, Solal AC, Joubert D, Legraverend C. Visualization of S100B-positive neurons and glia in the central nervous system of EGFP transgenic mice. J Comp Neurol. 2003:457(4):404-19.

34. Hachem S, Aguirre A, Vives V, Marks A, Gallo V, Legraverend C. Spatial and temporal expression of S100B in cells of oligodendrocyte lineage. Glia. 2005;51(2):81-97.

35. Buffo A, Vosko MR, Ertürk D, Hamann GF, Jucker M, Rowitch D, Götz M. Expression pattern of the transcription factor Olig2 in response to brain injuries: implications for neuronal repair. Proc Natl Acad Sci USA 2005;102(50):18183.

36. Kronenberg G, Gertz K, Cheung G, Buffo A, Kettenmann H, Gotz M, Endres M. Modulation of fate determinants Olig2 and Pax6 in resident glia evokes spiking neuroblasts in a model of mild brain ischemia. Stroke. 2010:41(12):2944-9.

\section{Publisher's Note}

Springer Nature remains neutral with regard to jurisdictional claims in published maps and institutional affiliations. 